\title{
Treating combat related PTSD and combat operational stress reaction a clinician's review
}

\author{
Volume 7 Issue I - 2017 \\ Jadidi $M$
}

Keywords: PTSD, COSR, combat operational stress reaction, barriers-obstacles to treatment, bio-psycho-social symptomology

Abbreviations: VA, veterans administration; CDP, center for deployment psychology; CPT, cognitive processing therapy; COSR, combat operational stress reaction; PTSD, post traumatic stress disorder

\section{Introduction}

There is a foundation of past and current research, as well as current ongoing research related to PTSD and COSR. Much of this research has been done by the Veterans Administration (VA), as well as by the Center for Deployment Psychology (CDP). Additionally, there has been research done related to best practice evidenced based treatment. There are two types of treatment modalities that have shown good outcomes and are practiced within the VA and military systems. These are (CPT) Cognitive Processing Therapy and (PE) Prolonged Exposure Therapy. In addition, there is an increasing amount of private practice clinicians that are also being trained and practice these types of therapies. There are also many other treatment modalities that are also being researched related treating these conditions. In addition, a considerable amount of time and research has gone into reflecting on the probable causes of treatment dropout rates.In my practice, along with the practice of my colleague's that I have consulted, the biggest cause of dropout rates, as well as with poor outcomes is related to identifiable treatment obstacles-barriers. In my practice, I have identified a baseline list of these obstaclesbarriers. These will be the main focus of this paper because if clinicians do not become proficient in identifying and addressing these obstacles-barriers treatment outcomes will be poor regardless of the treatment modality. In addition, in many cases, what is seen as obstacles are, in fact, Bio-Psycho-Social symptoms directly related to Combat Operational Stress Reaction (COSR) and Combat related Post Traumatic Stress Disorder (PTSD). ${ }^{1-4}$

To start to gain a clear understanding lets first start with defining Stress, Combat Operational Stress Reaction and Combat related Post Traumatic Stress Disorder. My definition of stress is any situation(s), event, observation, news, behavior, or thought(s) that creates and/or increases one's emotional discomfort and directly effects one's sense of control, sense of feeling safe, and sense of emotional stability. Combat Operational Stress Reaction is not a DSM mental health diagnosis. It is a military term used to describe the stress reaction service members can have related to combat operations. Although, in many cases, if this stress reaction is severe enough the military member could fit criteria for Acute Stress Disorder and if symptoms continue for more than 30days and/or increase the service member could then meet criteria for PTSD. Please see the DSM-5 for specific criteria for diagnosing Acute Stress Disorder and Post Traumatic Stress Disorder. "PTSD can occur after someone goes through, sees, or learns about a traumatic event like combat exposure, sexual or physical abuse/assault, terrorist attack, series accident or natural disaster." (National Center for

PTSD) The Center of Deployment Psychology describes Trauma as, "Trauma is the physical and emotional reaction to an event(s) that one perceives as life threatening and/or emotionally overwhelming, as well as feeling that one has little to no control over the situation and/or outcome of the situation." To recap, Combat Operational Stress Reaction and Combat related trauma can be linked progressively to Acute Stress Disorder and Post Traumatic Stress Disorder. In many cases, long term/chronic Combat related PTSD can increase the change developing a major depressive episode. Research indicates that a combination of therapy and mental health medications works best to treat combat related PTSD.

As stated in the introduction, my focus is going to be on known Bio-Psycho-Social social barriers that complicate and can influence outcomes and dropout rates. These barriers also in many cases influence military members and veterans to not seek treatment. A significant percentage of medical and mental health professionals' are familiar with the foundation symptoms of Combat Operational Stress reaction and Post Traumatic Stress Disorder. To assure that everyone reading this paper will have a baseline understanding, I will describe these symptoms. Baseline symptoms of Combat Operational Stress Reaction are usually an initial feeling of shock-dazed and maybe somewhat confused. The individual will have a hard time calming down and will normally have an increased startle response. The individual will normally feel an increase in agitation and/or anxiety. Many times there will be a sense of not feeling totally safe. There can also be issues with nightmares, overall sleep disturbance and on occasion flashbacks and or intrusive thoughts; although, the last two symptoms are more common when the condition progresses into clinically diagnosed PTSD. In many cases, these symptoms will dissipate after a few days. If these symptoms do not dissipate and instead they continue they can eventually transition into PTSD. Baseline symptoms of PTSD are very similar to the COSR, but usually more severe. Sometimes the military member will have these initial symptoms and then for multiple reasons internalize the symptoms and then they can re-surface in the future-months and sometimes years 
down the road. In addition to the above symptoms baseline Combat related PTSD can include reliving the event/s through flashbacks and/or intrusive thoughts (a type of dissociate episodes). These can be brought on by triggers such as visual, auditory or by certain smells. Additional symptoms may include not feeling safe, becoming emotionally distant/detached, anger, anxiety, problems focusing/ concentrating, avoidance, as well as issues with trust. Let's start to differentiate and break down the barriers/hurdles.

To begin with, as stated in the previous paragraph, PTSD/trauma symptoms can sometimes, for multiple reasons not be addressed and instead they get internalized and blocked from one's memory. When this happens, there will still be some symptoms including but not limited to anger issues (emotionally reactive), trust issues, degrees of anxiety, sadness, sleep disturbance, isolating and emotional detachment. When this happens the individual will in many cases be initially misdiagnosed. Additionally the service member could be experiencing unexplained physical symptoms. An example of this is the individual could be having physical discomfort or pain somewhere in their body for no apparent reason, when in actuality the pain mirrors where they had gotten hurt during the trauma. It does not get connected to the trauma because the memory of the trauma (or at least part of it) has been blocked. Some research indicates that traumatic events or parts of the events never get processed into long term memory. This is important to note because during treatment the therapist might be trying to help the military member process part of a memory that is not even actually there. Additionally, when faced with a traumatic event, a human being is in not seeing the event 100 percent accurately because the brain will click into the fight or flight mode, resulting in blood flow in the brain being shifted. Blood flow is taken away from the part of the brain that is able to accurately process the given situation. At that point, survival becomes a priority for the brain on an instinctual level. Internalizing/blocking the traumatic event can be a barrier for multiple reasons. First, the individual might be getting therapy for something else, but the trauma gets triggered resulting in multiple new and in some cases life threatening symptoms. At that point the therapist might not be trained in how to address or even properly diagnose what is going on. PTSD symptoms can be triggered months if not years after the actual traumatic event/s. Given this fact, unfortunately, many times when PTSD is triggered after years of being blocked certain providers or therapists don't believe it could be PTSD after all those years, when in fact it is. As a clinician or provider, knowledge related to combat related PTSD, consulting peers, ruling out other diagnoses and giving yourself time to think things through will aid in making a correct diagnosis.

The next two possible barriers/hurdles are also PTSD symptoms, as well as defense mechanisms. These are avoidance and anger. At this point, I would like to point out that trauma and PTSD symptoms are incredibly painful, so when I say anger and avoidance are also a defense mechanism it is because of that intense ongoing emotional pain and for the most part acted out automatically on subconscious level. Let's first reflect on anger. Excessive anger can of course be associated with many mental health diagnosis; for example anger can be a symptom of depression or anxiety or just of excessive stress. When related to a PTSD diagnosis, anger can affect every part of the individual's life. It can affect relationships, work, social situations, as well as one's physical health. An example of this would be a military member coming back from a deployment and becoming progressively angry and disillusioned related their military job or even their civilian job. If not recognized as a symptom of PTSD, they could end up leaving or getting fired from that job or even ongoing multiple jobs. Another example is when someone is having anger issues for any reason, we have the tendencies of projecting that anger into relationships with people we are the closest to and around the most which can lead to things like marital issues. Again, if it is not known that the anger is related to PTSD it can lead to many unnecessary negative consequences and outcomes.

Avoidance is a bench mark symptom of combat related PTSD, as well as can be a barrier/hurdle to treating it successfully. Avoiding will normally start with little things like avoiding talking about certain issues that manifest certain feelings. Remember, with this diagnosis comes extreme emotional pain. The avoidance can also develop with crowded places, as well as any people, places or things that could be related to or associated with the traumatic event. Progressively, the avoidance usually gets worse affecting every part of one's life. It can also lead and contribute to isolating. In regards to treating PTSD, individuals can become masters at avoidance when speaking. In turn, bringing up and processing issues can be challenging. Additionally, avoidance can contribute to dropout rates and/or not showing up for therapy appointments. If a clinician or provider doesn't recognize avoidance as a symptom of PTSD it can be perceived as noncompliance resulting in more treatment barriers. On the other hand when it is recognized as a symptom it can be handled as a symptom and addressed in the treatment process. Additionally, when addressing avoidance a sense of feeling safe is normally inter woven in it and needs to be addressed and processed. Cognitive Behavioral Therapy techniques and some types of processing therapies have proven to be effective when addressing anger and avoidance, and with avoidance components of exposure therapy are useful.

Take a minute and reflect on this question, "If you combine anger and avoidance to acting out behavior, how do you think that might impact someone's life?" Let's go back to the previous example of where a service member is returning from a deployment. Let's say that anger is causing issues with their job and in combination they are avoiding, by means of not showing up for work, not calling in, and or not returning calls. You can imagine the probable outcome and for the employer in most cases it is just seen as a behavioral problem and not recognized as a symptom of PTSD. Again, if avoidance and anger is recognized as a symptom it can be discussed/processed and a plan put in place to address it. ${ }^{5-8}$

Another significant symptom and hurdle is related to addressing trust issues. When someone experiences a trauma that leads to Combat Related PTSD their life is impacted to the degree where it is very difficult to trust others. This factor could negatively affect treatment or if addressed correctly, it could be used as a major part of the healing process. In addition to trust related issues, in many cases military members returning from deployments will experience difficulty with emotional intimacy with their love ones. Due to this fact, it is very important to develop a slow therapeutic relationship. The military member needs to know you are non-judgmental, that you have knowledge and experience working with military members and PTSD, and that you will be able to listen to their story and not break down.

In many cases, what military members experience while deployed is intense and can elicit a strong emotional reaction in other when heard. As a clinician or provider it's important to listen with empathy and understanding while maintaining your composure. While working with patients with a PTSD diagnosis not related to combat, if the therapist gets tearful and/or emotionally shaken when hearing a story that can actually help build a bond with a patient, but not so much with a military member or veteran. If the clinician gets emotionally shaken and/or tearful, the military member will most likely shut down 
and not go on with their story and not for the reason you might think. They will shut down in an attempt to protect you from their pain. To the military member, in many cases, it is an indication that you can't handle their story and there by not be able to help them. Service members and veterans will also most likely shut down if the clinician starts to ask questions that appear judgmental. The service member is first looking for someone to listen to their story and initially with little feedback other than supportive statements. Please remember there are two goals here. One is to help the service member make sense of how they are feeling and why are they feeling that way, but just as important that they can start to develop a trusting relationship with another person. This is called a learned emotional experience. This trust will allow you to help the military member using the chosen treatment modality.

As a therapist/clinical social worker, I use to work in the prison system. Every day when I came in and left, I had to go through a metal detector. When talking with the corrections officers, I found out the metal detectors can be set to different levels (sensitivity level). That can be compared to what happens to military members when deployed. As a human being, survival is one of the things that is hardwired into the human brain. That is regulated in the Limbic System (fight, flight or freeze response). This system can become more sensitive (turned up like the metal detector) depending on the threat level. If this system is turned up, the person will react quicker to environmental stimuli. One becomes more hyper vigilant. That's one of the reasons why people coming back from deployments need time to readjust and to readjust that fight or flight survival response. Sometimes when an individual is exposed to high threat levels for long periods of time and when combat trauma is present certain parts of the Limbic System gets damaged. There is a lot of research being done related to this, but what I have not seen with research is, if given the appropriate treatment can the Limbic System go back to normal. I have seen military get better with reduced symptoms to the point where they don't even meet criteria for PTSD. This is very promising and a good indicator that the brains Limbic System can heal itself, but I have not, as of yet seen the research to support that yet. In previous paragraphs, I was talking about anger, which also is linked to the Limbic system one's fight or flight threat response.

There are many other possible barriers, such as guilt, remorse, resentments, and the grieving process. These can all be more effectively addressed once the above for mentioned treatment hurdles are addressed. The research indicates that a combination of both mental health medications and therapy in combination is the most effective. When a military member has experienced a combat related trauma/s, and then develops PTSD it can be described as being stuck in that trauma. Many people know what is like to be stuck in emotional pain related to the grieving process, but in addition to that, having combat related PTSD can be like being stuck in the worse nightmare imaginable. That is why in many cases, a secondary diagnosis can develop related to depression and/or anxiety. The healing process related to PTSD can at times be a long process, but with the correct treatment, I have seen great progress and healing. Some of these hurdles mentioned need to be addressed at the very beginning of treatment such as developing trust, but even then you continue to work with your patients throughout the entire process related to these defined hurdles. There is a lot of controversy related to what treatment modality to use with combat related PTSD. It is my suggestion to stick with research and suggestions put forth by VA and the Center for Deployment Psychology (CDP). They are the experts in that field and have developed to date the most effective evidenced based treatment, such as Cognitive Processing Therapy and Prolonged Exposure Therapy.

\section{Acknowledgments}

None.

\section{Conflicts of interest}

Author declares there are no conflicts of interest.

\section{Funding}

None.

\section{References}

1. Carr Russell B. Combat and Human Existence: Toward an Intersubjective Approach to Combat-Related PTSD. Psychoanalytic Psychology. 2011; 28(4): 471-496.

2. (CDP) Center for Deployment Psychology Training for CPT and PE/ Star behavioral Health.

3. PTSD: National Center for PTSD. Health Care, USA. 2016.

4. Bremner JD. Traumatic stress: effects on the brain. Dialogues Clin Neurossci. 2006;8(4):445-461.

5. Https://sycofx.wordpress.com/2011/08/16/happiness-and-theamygdala/

6. DODSER. Calendar Year 2014 Annual Report, USA. 2004.

7. Goodwin RD, Cohen GH, Tamburrino M, et al. Mental health service use in a representative sample of national guard soldiers. Psychiatr Serv. 2014;65(11):1347-1353.

8. Rhoton Robert. Trauma, PTSD and Traumatic Grief. PESI, USA. 2015. 